\title{
Recombination, Ionization, and Nonequilibrium Electrical Conductivity in Seeded Plasmas
}

\author{
Trerrill A. Cool* and Edward E. Zukoski \\ Daniel and Florence Guggenheim Jet Propulsion Center, California Institute of Technology, Pasadena, California
}

(Received 16 September 1965)

\begin{abstract}
New data are presented which provide direct experimental confirmation of the validity of a physical model which has been widely employed to predict the electrical conductivity of dense, two-temperature, seeded plasmas. Experimental measurements of electron temperature, and ionization and recombination rates are presented for partially ionized plasmas of potassium-seeded argon. Experimental conditions were chosen to cover those ranges of interest in connection with proposed magnetohydrodynamic energy conversion devices for which nonequilibrium electrical conductivity measurements have been previously reported, e.g., translational atom temperatures of about $2000^{\circ} \mathrm{K}$, total atom densities near $10^{18} / \mathrm{cm}^{3}$, potassium densities of about $10^{16} / \mathrm{cm}^{3}$, electron densities from $10^{13} / \mathrm{cm}^{3}$ to $10^{15} / \mathrm{cm}^{3}$, and electron temperatures from 2200 to $3500^{\circ} \mathrm{K}$. Measured values of electron-electron-ion recombination coefficients for potassium show good agreement with theoretical values based upon the Gryzinski classical inelastic-collision cross-section expressions. Observed ionization rates and relaxation characteristics appear to be adequately explained by a similar formulation for the ionization process.
\end{abstract}

\section{INTRODUCTION}

$\mathrm{T}$ HERE has been considerable recent interest in the use of nonequilibrium effects to obtain enhanced electrical conductivity at moderate translational gas temperatures in magnetohydrodynamic devices. Much of this interest stems from the original work of Kerrebrock and others, ${ }^{1-5}$ who investigated the behavior of the two-temperature plasma which occurs in dense, alkali-vapor-seeded, noble gases when the electron temperature is elevated above the translational gas temperature due to the acceleration of electrons by an applied electric field. More recently, Kerrebrock and Hoffman ${ }^{6}$ and the present authors $^{7,8}$ have independently obtained experimental measurements of nonequilibrium electrical conductivity in seeded plasmas which show substantial agreement with predicted values based upon theoretical formulations utilizing the simple twotemperature plasma model proposed by Kerrebrock. ${ }^{1}$ This careful experimental work has led to several modifications of Kerrebrock's original theory, the most significant of which has been the calculation of the influence of inelastic collision energy losses

\footnotetext{
* Present address: Cornell University, Ithaca, New York.

1 J. L. Kerrebrock, in Engineering Aspects of Magnetohydrodynamics (Columbia University Press, New York, 1962), p. 327.

2 D. J. BenDaniel and S. Tamor, General Electric Technical Information Series 62-RL-(2922E) (1962).

3 F. Robben, Phys. Fluids 5, 1308 (1962).

4 W. Westendorp, C. Bishop, H. Hurwitz, L. Goldman, and D. BenDaniel, Phys. Fluids 4, 786 (1961).

5 D. BenDaniel and C. Bishop, Phys. Fluids 6, 300 (1963)

6 J. Kerrebrock and M. Hoffman, AIAA J. 2, 1072 (1965)

7 E. E. Zukoski, T. A. Cool, and E. G. Gibson, AIAA J. 2, 1410 (1964)
}

${ }^{8}$ E. E. Zukoski and T. A. Cool, AIAA J. 3, 370 (1965). from the free electron gas. Following a suggestion due to Kerrebrock, ${ }^{6}$ this net rate of energy loss from the free electrons due to inelastic collisions was estimated by detailed approximate calculations of plasma radiation losses. The resulting comparison of measured conductivity values and theory has been quite satisfactory over a wide range of experimental parameters, including variations in gas temperature, seed concentration, atomic cross sections, current density, electric field strength, degree of ionization, and energy loss processes. ${ }^{7-9}$ The revised two-temperature theory thus appears to give a useful solution to the problem of the calculation of nonequilibrium electrical conductivities in steady-state two-temperature plasmas.

There have been, however, some important and interesting unanswered questions concerning the validity of the simplified physical plasma model that has been employed in these studies. The most easily measured characteristic of the plasma is the electrical conductivity, and this is the quantity that has been studied most thoroughly. Though these measurements show good agreement with theory, this fact is necessary but not sufficient to establish the validity of the key assumption made in the twotemperature plasma model: namely, that the populations of the excited states and the density of the free electrons are assumed to be defined by the condition of thermal equilibrium at the electron temperature. It may be shown, in fact, that even if the actual electron density were significantly less than that corresponding to equilibrium at the elec-

\footnotetext{
${ }^{9}$ J. Kerrebrock, AIAA J. 3, 591 (1965).
} 
tron temperature, there would be no detectable difference in the nonequilibrium conductivity characteristics that have been previously measured. ${ }^{10}$ One purpose of this paper is to demonstrate that the assumption of ionizational equilibrium at the electron temperature is valid over a wide experimental region and, therefore, that this simple physical model is reasonably accurate for the description of plasma properties under many conditions of practical interest.

In this paper, the assumptions that have been made in the theoretical formulations employing the two-temperature plasma model are briefly examined, and new experimental data are presented which provide a conclusive quantitative check upon the validity of this simple two-temperature plasma model. The region of validity of the model is demonstrated, and new data are presented which clarify previous experimental uncertainties and extend previous conductivity measurements to very low current densities for which the model is no longer valid.

Further, results are presented of experimental measurements of recombination rates, ionization rates, and electronic collisional relaxation characteristics of potassium-seeded plasmas obtained by the examination of plasma behavior in response to step increases and decreases in electric field strength. These measurements show that the ionizational and recombinational relaxations of these plasmas are adequately described by a theory which employs a simplified model for the relaxing plasma in terms of the recently developed Gryzinski classical electron-atom inelastic-collision cross-section expressions.

A simple theory for the ionizational relaxation process is presented which appears to adequately describe available experimental measurements. This analysis explains the observed qualitative behavior of the electron temperature during the ionizational relaxation period and indicates that physical assumptions made in an earlier analysis of reference ${ }^{6}$ are inappropriate for the present problem.

The measured values of the electron-electron-ion recombination coefficients for potassium are shown to agree satisfactorily with theory, as has previously been reported for cesium, helium, and hydrogen.

No effort has been made in this brief introduction to give an account of the historical development of this field of research. Rather, the contributions of individual authors are cited at the points where their technical contributions enter naturally into the

${ }^{10}$ T. A. Cool, Ph.D. thesis, California Institute of Technology (1965). logical development of this work. A comprehensive review of the subject of nonequilibrium ionization as related specifically to the problem of magnetohydrodynamic power conversion has been given by Kerrebrock. ${ }^{9}$ Following a brief description of experimental techniques in Pt. II of this paper, Pt. III outlines the approximations which enable description of plasma behavior with simple theoretical considerations. In Pt. IV the new experimental results concerning the validity of the steady state twotemperature plasma model are presented. In Pts. V and VI the experimental measurements of ionizational and recombinational relaxations are presented and compared with approximate theory.

\section{EXPERIMENTAL APPARATUS}

A detailed description of experimental techniques and of the apparatus employed in making the measurements to be discussed here has been presented in detail elsewhere. ${ }^{10}$ An arc-jet heater, shown schematically in Fig. 1, is used to heat the larger portion of an argon or helium carrier gas flow. This main flow is then combined with a smaller, secondary flow of argon or helium which has been saturated with potassium vapor by passing it through a potassium boiler. The combined flow then passes through a mixing chamber in which the flow is equilibrated to a uniform, homogeneous "seeded plasma" before entering the test section at atmospheric pressure. In order to utilize the sodium line reversal technique for temperature measurements, small quantities of sodium were placed with the potassium in the potassium boiler.

A typical test section is shown in Fig. 2 (dimensions are in inches). It was established that the electric field was constant along the duct by inserting as many as 6 probes into the flow. The average electrical conductivity was determined by the relation $\sigma=(I / A) /(\Delta V / l)$, where $\Delta V$ is the floating probe potential difference, $\ell$ is the probe separation, $I$ is the measured total current, and $A$ is the cross-sectional area of the duct.

Spectroscopic measurements were performed by viewing the plasma through the ports shown in Figs. 1 and 2. Care was taken to eliminate undesirable absorption effects caused by the effusion of potassium vapor from the ports by purging the ports with a small auxiliary flow of argon. The physical arrangement of the photomultiplier tubes, optical filters and the spectroscope used for light intensity measurements is shown in Fig. 1. The photomultiplier tubes and optical filters were selected to isolate the $4 p \rightarrow 4 s, 5 p \rightarrow 4 s$, and $6 s \rightarrow 4 p$ transitions 


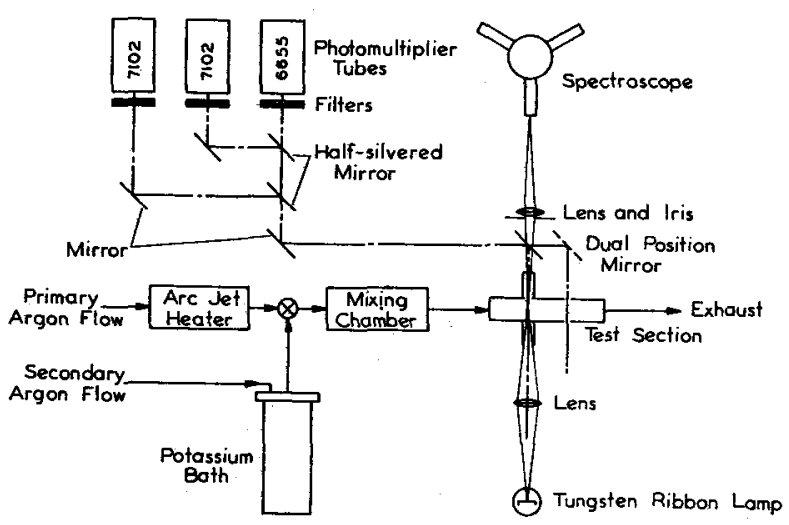

FIG. 1. Schematic diagram of apparatus.

in the line spectrum of $K_{1}$, and the $3 p \rightarrow 3 s$ transitions of $\mathrm{Na}_{\mathrm{I}}$. By rotating the light path to the photomultipliers out of the line of sight of the spectroscope, it was possible to make steady-state sodium line reversal measurements.

Figure 3 shows a schematic diagram of the electric circuit which enabled the study of plasma behavior either under the action of a steady electric field or during the relaxation periods immediately following an abrupt increase or reduction in electric field strength.

The various plasma properties measured experimentally are actually volume-averaged quantities as is evident from the above discussion. The approach used throughout this study has been to regard the quantities appearing in the various equations to be volume-averaged or bulk values instead of local values, and thus to interpret the equations as relations between volume-averaged rather than local quantities. The validity of such a uniform property analysis depends on how accurately one can characterize a given plasma property in terms of its

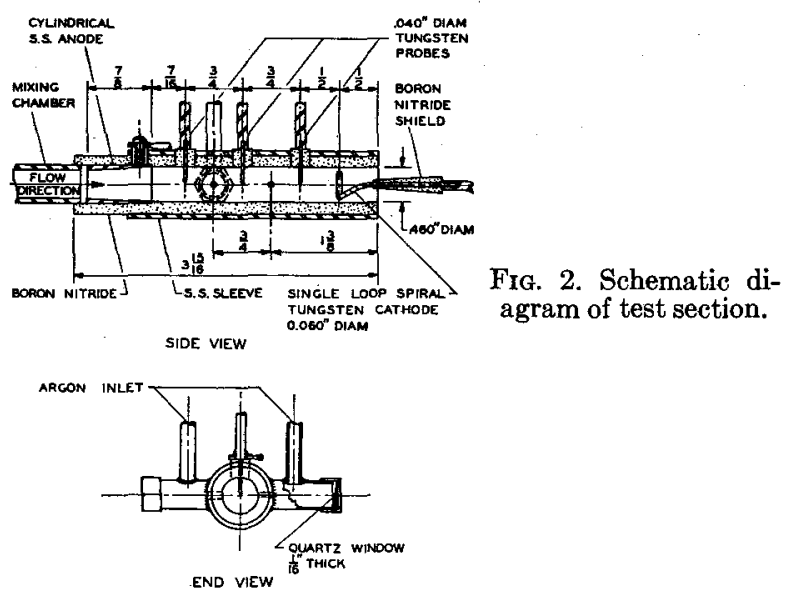

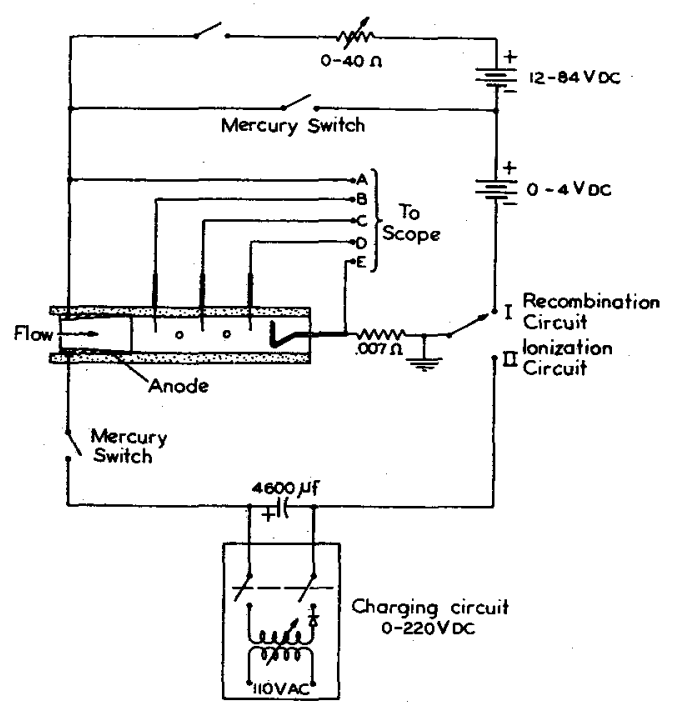

FIG. 3. Schematic diagram of electric circuit.

volume-averaged or bulk value, which one measures experimentally.

Measurements of the translational temperature profile for the atomic species indicated that the gas temperature could be accurately characterized in terms of its bulk value and that the gas temperature was within about $10 \%$ of this bulk value over nearly all of the cross section of the test duct. Energy balance considerations show that electron temperature variations are probably within the same limit. ${ }^{10}$ Electron density variations may be somewhat larger for regions less than about 1-mm from the walls.

\section{SIMPLIFIED PLASMA DESCRIPTION}

A description of plasma properties of the dense, seeded, monatomic plasmas of interest here would include a detailed knowledge of the populations of all bound electronic states, a knowledge of the translational energies of the free electrons and the various atomic species, and a determination of the free-electron density. In general, this formulation would involve the solution of the Boltzmann equation for each plasma species in terms of the various collisional interactions to specify the distribution functions of these various species. However, an extensive simplification results when the velocity distribution functions of the various plasma species are assumed to be Maxwellian to the first approximation. The establishment of the validity of this assumption requires an investigation of the influence of the various interactions between plasma species and the effects of the applied field upon the distribution functions of the various species. Calculations of this type indicate that at sufficiently high electron 
densities $\left(N_{e}>10^{13} / \mathrm{cm}^{3}\right.$ for conditions of these experiments), mutual electronic interactions are probabiy sufficiently frequent to "Maxwellianize" the electron distribution function by removing the effects of perturbations due to elastic and inelastic collisions with the various atomic species. ${ }^{2,6,10}$ Given this simplification, the description of properties would still require, in the general case, the simultaneous solution of the infinite set of differential equations determining the time variation of energy level populations, along with energy equations relating the instantaneous translational energies of the various species to the instantaneous energy level populations. ${ }^{11}$

Great simplifications result when the electron density is high enough so that the effects of electronic collisional processes dominate over those of atomic collisional and radiative processes in the rate equations which describe the populations of the free electrons and bound electronic states. Under these conditions, collisional equilibrium at the electron temperature prevails, and the free electron density at steady state may be related to the density of atoms in the ground state, for a given electron temperature, in terms of the Saha equation. Also, the transient problem is drastically simplified for this case and can be entirely formulated in terms of the recently developed Gryzinski electronic inelastic-collision cross-section expression $\mathrm{s}^{12}$ since only electronic collisional rate processes need be considered..$^{11,13} \mathrm{~A}$ short, informative discussion of these rate processes has been given by Robben. ${ }^{14}$

Additional simplification results because the ionization and excitation energies of the inert carrier gas are sufficiently large compared to those of the potassium seed that excitation and ionization of the carrier gas can be neglected at the electron temperatures considered here. The carrier gas thus acts only as a sink for the transfer of translational energy from the free electrons, as well as a barrier for diffusion of electrons and potassium ions. Note also that translational gas temperatures and carrier gas densities are assumed large enough to ensure that concentrations of the dimer form of potassium are negligible. ${ }^{15}$

These assumptions simplify the description of the plasma to a specification of the electron temperature

${ }^{11}$ D. R. Bates and A. E. Kingston, Proc. Roy. Soc. (London) A279, 10 (1964); A279, 32 (1964).

12 M. Gryzinski, Phys. Rev. 115, 374 (1959); 138, A305 (1965); 138, A336 (1965).

${ }_{13}$ D. R. Bates, A. E. Kingston, and R. W. P. McWhirter, Proc. Roy. Soc. (London) A267, 297 (1962); A279, 155 (1962).

${ }_{14}$ F. Robben, AIAA Preprint No. 64-56 (1964).

15 L. P. Harris, J. Appl. Phys. 36, 1543 (1965).
$T_{\text {e, }}$ the atomic species' translational temperature $T_{\mathrm{a}}$, the populations of the excited states for the potassium atoms $N_{i}$, and the number density of the free electrons $N_{\mathrm{e}}$. The plasma energy-transfer mechanisms which must be examined are the energy gain to the free electrons from the field, elastic and inelastic transfer of energy between the electrons and the various atomic species, as well as free-free, free-bound, and bound-bound radiative transfer.

\section{Electron Temperature}

Utilizing the foregoing simplifications, the electron temperature can be determined by considering the local energy balance for the free electrons under the application of an electric field $E$;

$$
\begin{aligned}
N_{\mathrm{e}} & \frac{d \epsilon_{\mathrm{e}}}{d t}=\sigma E^{2}-\dot{\Omega} \\
- & {\left[\left(\epsilon_{\mathrm{e}}+V_{0}\right) \frac{d N_{\mathrm{e}}}{d t}+\sum_{i} V_{i} \frac{d N_{i}}{d t}+\dot{R}\right] }
\end{aligned}
$$

the rate of change of the energy of the free electrons (per electron) per unit volume is written on the left-hand side of Eq. (1). Here $\epsilon_{\mathrm{e}}=\frac{3}{2} k T_{\mathrm{e}}$, where $k$ is the Boltzmann constant and the ionization potential of potassium is given by $V_{0}$ and is $4.34 \mathrm{eV}$. The rate of energy input per unit volume to the free electrons by acceleration in the electric field is given by $\sigma E^{2}$. Electrons lose energy by elastic collisions with the various neutral and ionized atomic species at a rate per unit volume given by $\dot{\Omega}$. Electrons transfer their kinetic energy into the creation of excited states in the seed atom at the net rate $\sum_{i} V_{i}\left(d N_{i} / d t\right)$ per unit volume, provided there are no radiative losses from the system. Here, $V_{i}$ is the energy, as measured from the ground state, of the $i$ th energy level with population $N_{i}$. For all systems of finite size, net radiative losses do occur, and so some of the energy transferred from the free electrons due to inelastic collisions which could otherwise appear as an increase in the density of excited and ionized states is instead lost from the system as radiation. For the conditions of these experiments, this radiative loss, $\dot{R}$, is principally that due to bound-bound transitions between excited states; the loss due to direct radiative recombination into excited states (free-bound transitions) and that due to free-free transitions (bremsstrahlung) being negligible by comparison.

If the electron energy can be regarded as quasisteady, then $N_{\mathrm{e}}\left(d \epsilon_{\mathrm{e}} / d t\right)$ is a small term compared to the others; also under some conditions, ${ }^{13}$ most of the $V_{i}\left(d N_{i} / d t\right)$ terms are also negligible, and then 
the populations of the corresponding excited states can be regarded as quasi-steady.

The key to simplification of the transient plasma description has been the recognition that many state populations in the relaxing plasma can be simply related by quasi-steady relations in terms of the instantaneous electron temperature and density. The result has been the reduction of the infinite set of coupled rate equations describing the time variation of energy-level populations to a finite set of algebraic equations describing the lower excited state populations, and an equation describing the rate of change of the free electrons and the ground state. ${ }^{13} \mathrm{~A}$ complete discussion of the requirements for these modifications is given by Bates et al. ${ }^{13}$. No details need be given here, but the physical reasoning is as follows. Immediately following a step change in the electric field strength applied to high density plasmas, the electron temperature quickly reaches a quasi-steady value given by the instantaneous energy balance on the free electrons. A similar, and in fact directly coupled, phenomenon exists with regard to the population densities of closely-spaced excited states in the relaxing plasma. The detailed classical formulation of electronic inelastic collisions indicates that the probability of a collisionallyinduced transition between two neighboring atomic levels is inversely proportional to the square of the energy gap separating them. ${ }^{16}$ Thus the populations of the closely-spaced upper levels near the continuum rapidly tend to approach a mutual quasi-steady collisional equilibrium with the free electrons, and in addition, closely-spaced lower levels reach a mutual quasi-steady relationship in times that are short compared to the time scale for changes in the populations of the free electrons and the ground state. This rapid establishment of quasi-steady conditions therefore allows a greatly simplified description of the overall transient relaxation.

Referring again to Eq. (1), a "steady-state" occurs when all the $d / d t$ terms are essentially zero and the whole system heats up relatively slowly as the energy is transferred into the translational energy of the atomic species. For this "steady-state" case we have

$$
\sigma E^{2}=J^{2} / \sigma=\dot{\Omega}+\dot{R}_{s} \text {. }
$$

Equation (2) gives the local electron energy balance of a two-temperature plasma under the influence of a steady electric field after the initial ionizational relaxation transients have vanished. This condition

${ }^{16}$ S. Byron, P. I. Bortz, and G. Russell, in Proceedings of the Fourth Symposium on Engineering Aspects of Magnetohydrodynamics (University of California Press, Berkeley, California, 1963). will be referred to throughout this paper as the "steady state."

As noted above, the energy balance equations can also be interpreted as relations between volumeaveraged quantities, provided that nonuniformities near plasma boundaries are not so large that the characterization of the plasma in terms of equations dealing with average quantities has no meaning. For the purpose of this analysis, the calculation of the detailed radiation loss from each local plasma element is not relevant, since we intend to characterize all experimental results by a bulk measurement of a given quantity and relate this experimental value to a theoretically calculated uniform quantity. Thus the total radiative loss from the plasma volume has been calculated assuming uniform properties, and then divided by the plasma volume to give the volume-averaged quantity $\dot{R}_{s}$.

\section{STEADY-STATE PLASMA}

The description of the steady-state plasma existing after the initial ionizational relaxation following the application of a steady electric field has been considered by several authors. ${ }^{1-5}$ The formulation discussed here and presented in detail elsewhere ${ }^{7,10}$ is based upon the earlier work of these several authors.

This steady-state model assumes electronic collisional processes are sufficient to ensure thermal equilibrium between the free electrons and the bound states of potassium and that the electron temperature defining this condition is given in terms of an electronic energy balance including both elastic and inelastic energy loss mechanisms from the free electrons, as was discussed in the preceding section.

The experimental results presented here and in Refs. 7 and 8 have led to several theoretical modifications which were found to be essential in accurately describing the observed plasma behavior. The elastic and inelastic losses are properly treated independently as two distinct physical processes by a formulation in terms of basic atomic properties which employs no adjustable parameters. The inelastic losses can be determined for the steady state by a detailed plasma radiative loss calculation." Additionally, consideration of the detailed energy dependence of the atomic cross sections and the inclusion of both electron-ion and electron-atom interactions has been essential in describing the experimental results. Finally, the electrical conductivity entering into this formulation is also described in terms of integral expressions over the electronic velocity distribution function and reciprocal sums of the various cross sections. The resultant conduc- 
tivity for the case of combined close and distant encounters is not exact, but the conventional reciprocal sum rule ${ }^{17}$ is felt to give a reasonable approximation to the conductivity over the range of experimental conditions.

\section{Steady-State Electrical Conductivity}

Extensive measurements of electrical conductivity were made at the steady-state immediately following the initial ionizational relaxation period after a step application of electric field. ${ }^{7,8,10}$ The ionizational relaxation was completed in times much shorter than the characteristic times for both the appreciable increase in the translational temperature of the atomic species and the flow of plasma through the test duct, and thus the plasma could be regarded as stationary with a constant atomic species translational temperature fixed by initial conditions. The experiments were performed at a pressure of one atmosphere and with translational atomic species temperatures varying over the range from 1250 to $2000^{\circ} \mathrm{K}$, current densities ranging between 0.4 and $90 \mathrm{~A} / \mathrm{cm}^{2}$, and for various potassium seed concentrations between 0.1 and 0.8 mole per cent. Seed concentrations for measurements in the argon-potassium plasmas were defined by the ratio $n_{\mathrm{K}} / n_{\mathrm{A}}$, specified for the initial plasma conditions with zero applied field. In addition, conductivity measurements were made in the helium-potassium plasma over a wide range of current densities at a single test condition.

The agreement between the calculated and measured values is illustrated by the data presented in Fig. 4. The data are in excellent agreement with the calculated values when the effects of radiant energy loss are properly included in the calculations and when current densities greater than $\frac{1}{2} \mathrm{~A} / \mathrm{cm}^{2}$ are considered. This agreement is typical of that obtained over the whole range of parameters for which data have been previously reported..$^{78}$ The good agreement obtained for gases with masses and electron-atom momentum transfer cross sections as different as argon and helium indicates that the general computational scheme is correct. In addition, note that at current densities near $1 \mathrm{~A} / \mathrm{cm}^{2}$ in the argon-potassium system, electron-ion interactions are negligible compared to electron-atom encounters; in contrast, at current densities near $80 \mathrm{~A} / \mathrm{cm}^{2}$, the Coulomb encounters are predominant. Thus, the good agreement between experiments and

${ }^{17}$ S. C. Lin, E. L. Resler, Jr., and A. Kantrowitz, J. Appl. Phys. 26, 95 (1955). theory indicates that the use of the Spitzer conductivity expression and the inclusion of electron-ion energy exchange in the theoretical calculations of Refs. 7 and 8 appears to be substantially correct.

Figure 4 shows data extending to low current densities in the range $0.025 \leq J \leq 0.40 \mathrm{~A} / \mathrm{cm}^{2}$. In this region the conductivity decreases with decreasing current density in a smooth way toward the equilibrium value. Earlier data, reported by Kerrebrock ${ }^{6}$ and the present authors, ${ }^{7}$ showed an anomalous behavior in that low current conductivity values reached a plateau at about $0.4 \mathrm{~A} / \mathrm{cm}^{2}$ and remained constant at about $0.2 \mathrm{mho} / \mathrm{cm}$ with decreasing current densities below $0.4 \mathrm{~A} / \mathrm{cm}^{2}$. This difficulty was investigated for the apparatus used in this study and was traced to the existence of a thin, poorly conducting film which builds up on the test section walls and which apparently shunts the voltage probes. This anomalous behavior was eliminated by testing with new boron nitride test section walls before this film appeared.

Low Current Density Data: The typical data shown in Fig. 4 illustrate that for very low current densities, below about $0.3 \mathrm{~A} / \mathrm{cm}^{2}$, the experimental values break away rather sharply from the theoretical curve which includes the radiative loss term in the electronic energy balance. The conductivity data show a smooth transition from this current density down to the lowest currents investigated $\left(\approx 0.02 \mathrm{~A} / \mathrm{cm}^{2}\right)$ and appear to level off at these lowest currents to a conductivity value approximately corresponding to the translational temperature of the gas, $T_{\mathrm{a}}=$ $2000 \pm 100^{\circ} \mathrm{K}$ for this case. A plausible explanation for this discrepancy between experiment and theory is the overestimation of the influence of radiation in the electron energy balance, caused by the neglect of the atom-atom excitational process and radiative

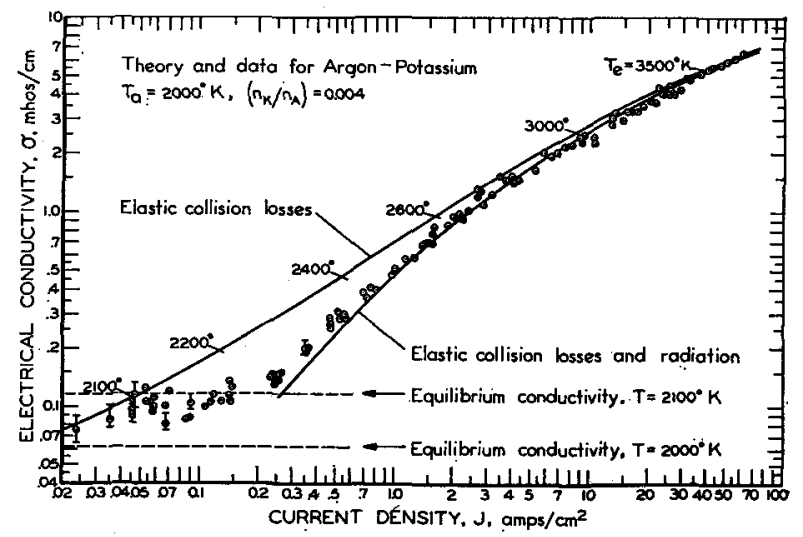

Fig. 4. Dependence of steady-state conductivity on current density. 
depopulation effects, both of which become important for low electron densities. ${ }^{10}$

Unstable Low Current Regions: Figure 5(a) shows the variation of electric field strength for the data of Fig. 4 as represented by the solid curve of Fig. 5(b). The current density and conductivity were observed to be quite sensitive to small changes in applied electric field strength for $0.4 \leq J \leq 1.5 \mathrm{~A} / \mathrm{cm}^{2}$. Similar unstable regions were observed at other gas temperatures and seed concentrations. In the unstable range, $d(\ln \sigma) / d(\ln J)$ is approximately unity, and consequently, the field strength is almost constant. However, if the radiation correction had been larger, as it would be in a smaller apparatus, the values of $d(\ln \sigma) / d(\ln J)$ would increase above unity, and thus the field strength would exhibit a local maximum in this region as is illustrated by the dashed curves of Figs. 5(a,b). Such a maximum was indeed observed at these low current densities by Kerrebrock and Hoffman, ${ }^{6}$ who employed a test section with a diameter about $60 \%$ of that used in the present experiments.

\section{Electron Temperature Measurements}

The good agreement between calculated and meas-
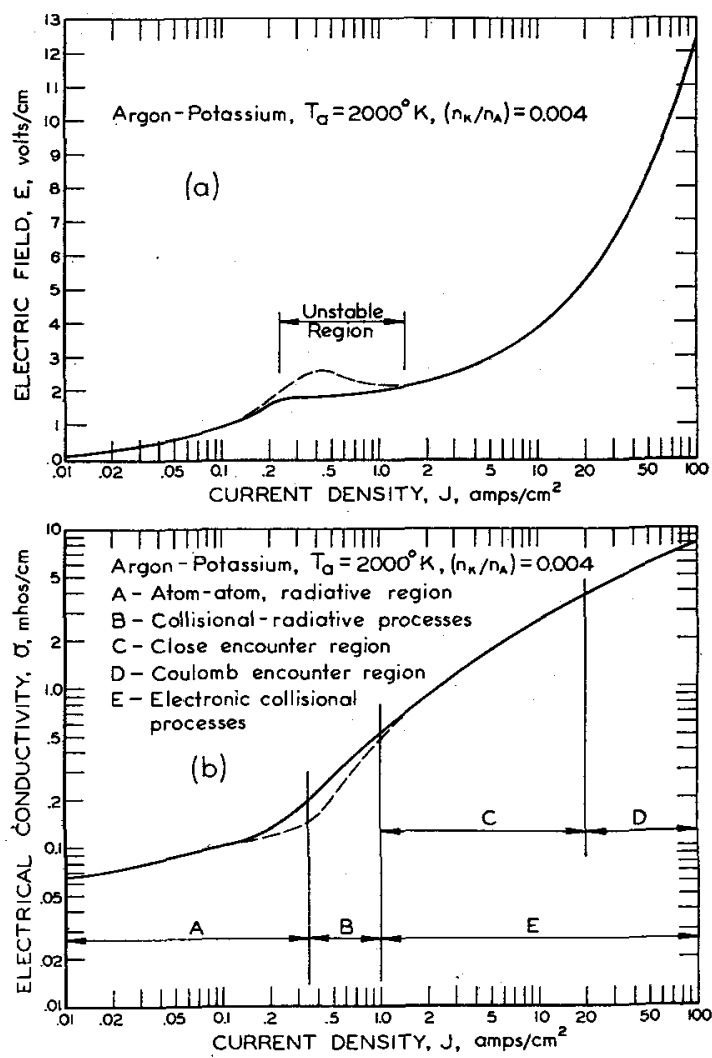

F1G. 5. Typical electric field and conductivity variations with current density. ured values of the electrical conductivity shows that the two-temperature model is useful in making conductivity estimates. This, however, does not provide a precise check of the key assumptions of the model. Calculations indicate that even if the electron density is actually significantly less than that corresponding to equilibrium at the electron temperature, no measureable difference in the curve of electrical conductivity versus current density is obtained. ${ }^{10} \mathrm{~A}$ good check can only be obtained by making a direct measurement of electron temperature and/or electron density. In this section, a description is given of the experiments used to obtain a measure of the electron temperature. The recombination experiments described in the following section were used to obtain estimates of electron density.

Two spectroscopic techniques were used to determine population temperatures of electronic states of neutral sodium and potassium atoms. (The population temperature of an observed state density in a system not necessarily in equilibrium is defined as the temperature one would associate with an equilibrium system possessing this same state density value.) In order to obtain the electron temperatures from these data, it is necessary to show that these states are in thermal equilibrium with the electron gas. Calculations for the argon-potassium system of the total probability of radiative decay, including the effects of absorption, were made for the lowlying levels of potassium to establish that for electron densities above $10^{14} / \mathrm{cm}^{3}$ the excited states and the electron gas are probably in equilibrium and, therefore, that population temperatures and electron temperatures are equal. ${ }^{10}$ For the argon-potassium system, calculated electron densities are greater than $10^{14} / \mathrm{cm}^{3}$ for current densities above about $2 \mathrm{~A} / \mathrm{cm}^{2}$. Calculations indicate that the equilibrium assumption becomes questionable for current densities below about $\frac{1}{2} \mathrm{~A} / \mathrm{cm}^{2}$.

The first spectroscopic technique employed was the well-known sodium-line reversal method, e.g., Ref. 18, which utilizes the $3 p \rightarrow 3 s$ transitions of $\mathrm{Na}_{\mathrm{I}}$ and provides an absolute measure of the population of the $3 p$ level of $\mathrm{Na}_{\mathrm{I}}$ and, hence, gives an absolute measure of the population temperature of the $3 p$ state. However, since the light source used in the experiments was a tungsten ribbon lamp, the measured values were limited to temperatures below about $2950^{\circ} \mathrm{K}$.

The second technique was a relative method by

18 A. Gaydon and H. Wolfhard, Flames, Their Structure, Radiation, and Temperature (Chapman and Hall Ltd., London,
1960). 
which measurements of light intensity from a given resonance transition made before and after the application of an electric field to the plasma were used to determine the change in the population of the upper state as a function of applied electric field and current density.

This method employed measured relative changes in light intensity to infer relative changes in upper state population temperature. For currents below about $30 \mathrm{~A} / \mathrm{cm}^{2}$, the line broadening by the Stark effect is negligible compared to that by collision broadening and Doppler broadening effects, and also the number density of atoms in the ground state remains essentially constant with changing current density. Thus, the absorption of a given resonance transition did not vary as a function of applied electric field or current density, and it was possible to infer the relative change in the density of atoms in the upper state of a given transition directly from the relative change in observed light intensity from that transition. The population temperature ratio can thus be directly calculated from the relation

$$
T_{1} / T_{2}=1-\left(k T_{1} / \Delta E_{i}\right) \ln \left(I_{2} / I_{1}\right) .
$$

Here, $I$ is the intensity of the $i$ th transition, $T$ is the temperature, and $\Delta E_{i}$ is the energy difference between the upper and ground states of the $i$ th resonance transition. The subscript 1 refers to conditions when the electric field is zero, and 2 to conditions when a field is applied.

Equation (3) was used to obtain temperatures from intensity data for the $3 p \rightarrow 3 s$ transitions in neutral sodium by the use of the $T_{1}$ value as obtained with sodium-line reversal measurement. These data and that obtained from the sodium-line reversal technique are shown as the solid points in Fig. 6 . The two sets of data agree reasonably well in the region between 6 and $8 \mathrm{~A} / \mathrm{cm}^{2}$, where they overlap. This indicates that the use of the relative method and Eq. (3) are consistent with the sodium-line reversal method and gives confidence in the relative method as it is used here.

In addition to the sodium measurements, data were obtained with two transitions in potassium. However, in order to use Eq. (3) with these data, it is necessary to determine the values of the temperatures in the zero field case. This is a nontrivial problem, because radiation losses keep the various excited states out of thermal equilibrium and because no absolute technique such as the sodium-line reversal method was readily available. (Reference 19 reports successful use of a potassium line reversal

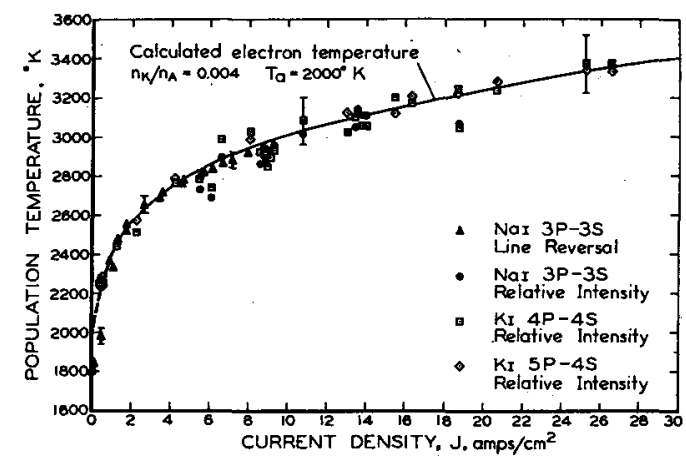

Fig. 6. Dependence of population temperatures on current density.

technique.) The method used to obtain the initial temperature was as follows. Relative intensity data were obtained simultaneously for the $3 p \rightarrow 3 s$ sodium transitions and the $4 p \rightarrow 4 s$ potassium transitions. The values of $T_{1}$ for the potassium transitions were picked to minimize the squared deviations between $T_{2}$ values calculated from Eq. (3) for the two transitions. (This calculation is based on the assumption that the various states will be in thermal equilibrium when the electron density is high.) In another set of experiments, simultaneous measurements of the intensities of the $4 p \rightarrow 4 s$ and $5 p \rightarrow 4 s$ potassium transitions were made. The value of $T_{1}$ for the $5 p \rightarrow 4 s$ transitions was picked to minimize the squared deviations between the two sets of data.

The zero field temperatures calculated by this technique for an $1800^{\circ} \mathrm{K}$ temperature of the $3 p \rightarrow 3 s$ sodium transitions were $1790^{\circ} \mathrm{K}$ for the $4 p \rightarrow 4 s$ potassium transitions and $1865^{\circ} \mathrm{K}$ for the $5 p \rightarrow 4 s$ potassium transitions. Use of these values of zero field population temperatures for the two potassium lines gives the values of population temperature shown in Fig. 6 as the open points. The data agree very well with each other and with the values obtained from the sodium data for the whole range of current densities investigated. This good agreement indicates that the equilibrium assumption made in interpreting the intensity data is justified.

In Fig. 6 brackets have been placed on some of the sodium-line reversal data and on some of the potassium data to indicate the maximum errors. The maximum uncertainty in the sodium-line reversal data is about $\pm 40^{\circ} \mathrm{K}$, and this uncertainty is due to errors in technique and drift in operating conditions for the arc-jet heater. The larger un-

${ }^{19}$ G. Brederlow, R. Hodgson, and W. Riedmüller, in Sixth Symposium on Engineering Aspects of Magnetohydrodynamics (1964). 
certainty for relative measurements reflects this $\pm 40^{\circ} \mathrm{K}$ maximum uncertainty in the zero-field sodium-line reversal temperature for these data.

Within the accuracy of the techniques, the initial population temperatures for the $\mathrm{Na}_{\mathrm{Y}} 3 p \rightarrow 3 s$ and $K_{\mathrm{I}} 4 p \rightarrow 4 s$ transitions are equal. The close agreement in these temperatures would be expected due to the similarity between the corresponding energy levels of the two atoms. However, the $65^{\circ} \mathrm{K}$ higher initial temperature for the $5 p \rightarrow 4 s \mathrm{~K}_{\mathrm{I}}$ transitions is significant within the capabilities of the technique. These measurements indicate the temperatures corresponding to these low lying states are about $200^{\circ} \mathrm{K}(4 p \rightarrow 4 s)$ and $135^{\circ} \mathrm{K}(5 p \rightarrow 4 s)$ below the mean translational gas temperature of $2000 \pm 100^{\circ} \mathrm{K}$. These differences indicate a lack of thermal equilibrium in the zero field case.

The solid curve shown in Fig. 6 represents the calculated value of electron temperature for the experimental conditions. Although the data are somewhat scattered, they clearly fall along the curve and, hence, are in excellent agreement with the calculated values. It is evident that the calculation gives a satisfactory estimate of electron temperature except at very low current densities.

Summary of Steady-State Measurements: The agreement between experimentally measured values of electron temperature and electrical conductivity with values calculated from the two temperature model presented in Refs. 7 and 10 is excellent over a wide range of experimental parameters and experimental conditions. It can be concluded that in this extensive region of agreement the two temperature model gives an accurate description of the plasma, and further that the key assumption of electronic collisional equilibrium is valid. The model is not adequate, however, for low electron densities (current densities below about $0.4 \mathrm{~A} / \mathrm{cm}^{2}$ ) where radiative depopulation effects and atom-atom excitation mechanisms appear to be important. Energy losses from the free electrons due to inelastic collisions have been shown to be an important effect for the small scale experiments discussed here.

This good agreement demonstrates the utility of this formulation in calculating nonequilibrium electrical conductivities for practical engineering systems utilizing monatomic gas-alkali vapor plasmas. The existence of the potentially large enhancement in ionization caused by electronic collisional processes has been conclusively demonstrated experimentally over the wide range of conditions investigated here. Note that, for example, the electrical conductivity at $10 \mathrm{~A} / \mathrm{cm}^{2}$ in the argon-potassium system for
$T_{\mathrm{a}}=1500^{\circ} \mathrm{K}, n_{\mathrm{K}} / n_{\mathrm{A}}=0.002$ (see Refs. 7 and 8 ) is about 2500 times the value one would calculate for equilibrium at the gas temperature.

\section{RECOMBINATIONAL AND IONIZATIONAL RELAXATIONS}

\section{Recombination}

Extensive theoretical recombination formulations have been performed utilizing a model which includes recombination to excited levels by electron-electronion collisions, electronic collisionally-induced transitions between excited levels, as well as the radiative transitions between bound states. ${ }^{1320,21}$ Careful experimental work has established remarkably good agreement between experimental results and theory for such differing atomic species as hydrogen, helium, and cesium. ${ }^{13,15,20,22,23,24}$ Bates et al. ${ }^{13}$ have performed detailed calculations for hydrogen and helium, and the resulting rates and population densities have been confirmed experimentally by several authors. ${ }^{20,22,24}$ In addition, detailed theoretical calculations have been performed giving recombination rates for argon, cesium, and potassium. ${ }^{16,23}$

Byron et al. ${ }^{16,21}$ have shown that good results can be obtained with fairly simple calculations by treating the collisional recombination processes as a chain of reactions in which the net rate of recombination can be described in terms of the slowest step in the chain. The physical picture consists of a reservoir of upper excited states in quasi-steady collisional equilibrium with the free electrons above a critical energy gap across which recombining atoms must pass to reach the ground state. The critical energy gap will be the one of all possible energy gaps across which the total deexcitation rate is a minimum. A minimum occurs, since the probability of deexcitation of a given level will increase with increasing principal quantum number of the level due to more closely spaced gaps, while the populations of the various levels decrease with increasing quantum number. In addition, the total probability of radiative decay of a given level decreases with increasing quantum number.

The Byron formulation has the powerful advantage of being easily applied to atoms with arbitrary

\footnotetext{
${ }^{20}$ E. Hinnov and J. G. Hirschberg, Phys. Rev. 125, 795 (1962).

${ }^{21}$ S. Byron, R. C. Stabler, and P. I. Bortz, Phys. Rev. Letters 8, 376 (1962).

${ }_{22}$ F. Robben, W. B. Kunkel, and L. Talbot, Phys. Rev. 132, 2363 (1963).

${ }_{23}^{3}$ J. V. Dugan, Jr., NASA Technical Note D-2004 (1964).

${ }^{24}$ R. W. Motley and A. F. Kuckes, in Proceedings of the Fifth International Conference on Ionization Phenomena in Gases (North-Holland Publishing Company, Amsterdam, 1961).
} 
energy level structures which are not necessarily hydrogenic. This calculation technique has been used here to obtain recombination rates for potassium in the range of interest of these experiments which occur at slightly higher electron temperatures than the maximum temperatures in the published results of Byron et al. ${ }^{16}$ As justified in Ref. 16, the rate of crossings in the upward direction across an energy gap $U$ located above a level of binding energy $E_{k}$ and coming from all initial energy levels with binding energies $E_{i}\left(E_{i} \geq E_{k}\right)$, to all possible final bound energy states as well as the continuum can be written as ${ }^{16,10}$

$$
\begin{aligned}
& \left.\frac{d N}{d t}\right|_{u}=N_{\mathrm{e}}\left\{\frac{N_{k}}{g_{k}} e^{-U / k r_{\bullet}}\right\} \bar{c} \sigma_{0} \\
& \cdot \sum_{i} \frac{B_{i} g_{i}}{\left(U+E_{i}-E_{k}\right)^{2}}\left(1+\frac{2 k T_{e}}{U+E_{i}-E_{k}}\right),
\end{aligned}
$$

where $B_{i}=B_{i}\left\{E_{i} /\left(E_{i}-E_{k}+U\right)\right\}$ is the slope of the linear portion of the Gryzinski cross section as given in Ref. $16 ; \sigma_{0}=6.56 \times 10^{-14} \mathrm{~cm}^{2} \mathrm{eV}^{2} ; \bar{c}$ is the mean thermal speed of electrons, $\left(8 k T_{\mathrm{e}} / \pi m_{\mathrm{e}}\right)^{\frac{1}{2}} . N_{k}$ and $g_{k}$ are the number density and degeneracy, respectively, of the energy level with binding energy $E_{k} . N_{\mathrm{e}}$ and $T_{\mathrm{e}}$ are the free electron density and temperature, respectively. The summation is to be taken over all energy levels below the gap $\left(E_{i} \geq E_{k}\right)$.

At equilibrium, the total rate of crossings of the gap in the upward direction must balance the total rate of crossings in the downward direction. Also, at equilibrium, $N_{\ell} / N_{k}=\left(g_{\ell} e^{-U / k T_{e}}\right) / g_{k}$, where $N_{\ell}$ and $g_{\ell}$ are the number density and degeneracy, respectively, of the energy level with binding energy $E_{k}-U$ located immediately above the gap. Thus, at equilibrium,

$$
\begin{gathered}
\left.\frac{d N}{d t}\right|_{d}=\left(\frac{N_{l_{\text {equi } 1}}}{g_{l}}\right) N_{e} \bar{c} \sigma_{0} \\
\cdot \sum_{i} \frac{B_{i} g_{i}}{\left(U+E_{i}-E_{k}\right)^{2}}\left(1+\frac{2 k T_{\mathrm{e}}}{U+E_{i}-E_{k}}\right), \\
N_{l_{\text {oqu } \mathrm{i} 1}}=\frac{g_{l}}{g_{\mathrm{e}} g_{\mathrm{ion}}}\left(\frac{h^{2}}{2 \pi m_{\mathrm{e}} k T_{\mathrm{e}}}\right)^{\frac{3}{2}} e^{+E_{l} / k T_{\mathrm{e}}} N_{\mathrm{e}}^{2}
\end{gathered}
$$

where $g_{\mathrm{e}}$ and $g_{\text {ion }}$ are the degeneracies of the electron and ion, respectively, and $h$ is Planck's constant.

The minimum value of the total rate of downward crossings, $d N /\left.d t\right|_{d \min }$, found by calculation from Eq. (5) for each energy gap, can be related to the recombination rate by the relation

$$
d N_{\mathrm{e}} / d t=\gamma d N /\left.d t\right|_{d \min },
$$

where $\gamma$ lies between $\frac{1}{4}$ and 1 . For the temperature range of these experiments, $\gamma \approx \frac{1}{3} .{ }^{16,21}$ A more complete discussion of the approximations implicit in Eq. (7) is given in Refs. 16, 21, 23.

Calculations of $d N /\left.d t\right|_{d \min }$ were performed for the low-lying levels of potassium to establish that, for electron temperatures between 2000 and $3000^{\circ} \mathrm{K}$, the minimum deexcitation rate occurs for the gap lying below the lumped $6 s$ and $4 d$ levels and above the $5 p$ level. Resulting values of the recombination rate coefficient, $\left(-1 / N_{\mathrm{e}}^{3}\right)\left(d N_{\mathrm{e}} / d t\right)$, calculated with $\gamma=\frac{1}{3}$ for $2000^{\circ} \leq T_{\mathrm{e}} \leq 3000^{\circ} \mathrm{K}$ are shown in Fig. 10. The recombination rate coefficient calculated at $2000^{\circ} \mathrm{K}$ agrees well with the value at $2000^{\circ} \mathrm{K}$ in Ref. 16. Also shown in Fig. 10 are the values for cesium as calculated in a more exact fashion with an extensive program by Dugan. ${ }^{23}$ Note that the calculated rates for cesium and potassium are nearly equal, which would be expected judging from the similarity of their energy level structures.

At no point in the foregoing calculations have the effects of radiative transitions upon the recombination rate appeared explicitly. Such radiative transitions can cause nonequilibrium populations in the levels immediately above the gap. This effect can be considered in the evaluation of the factor $\gamma$ in Eq. (7). One must still include, however, the possibility of direct radiative recombination when calculating the total rate of recombination. For the conditions of these experiments, direct radiative recombination is negligible, however. ${ }^{20}$

The effects of diffusion of ions to the walls as well as recombination by the dissociation reaction $\mathrm{K}+\mathrm{K} \rightleftharpoons \mathrm{K}_{2}^{+}+e^{-}$can be shown to be neglible for the densities and temperatures of these experiments $^{10}$ as was done by Harris for recombination experiments in cesium. ${ }^{15}$ Finally, the inert gas is assumed to play no appreciable role in the ionization and recombination processes studied here due to its relatively high ionization potential and the relative inefficiency of atom-atom excitations and ionizations.

Energy Balance for Recombination: As has been discussed, the energy of the free electrons can be regarded as quasi-steady, after a short initial transient, since the free electrons can exchange energy with the atomic species by elastic and inelastic collisions in times much shorter than those associated with changes in the population of the free electrons. The quasi-steady energy balance is given from Eq. (1) as

$$
\begin{gathered}
N_{\mathrm{e}}\left(\frac{d \epsilon_{\mathrm{e}}}{d t} \rightarrow \approx 0\right)=\sigma E^{2}+\left\{\left[-\left(\epsilon_{\mathrm{e}}+V_{0}\right) \frac{d N_{\mathrm{e}}}{d t}\right.\right. \\
\left.\left.-\sum_{i} V_{i}\left(\frac{d N_{i}}{d t} \rightarrow \approx 0\right)\right]-\dot{R}\right\}-\dot{\Omega}
\end{gathered}
$$


where, in addition to the assumption of a quasisteady electron energy, the populations of the excited states are also regarded as quasi-steady (i.e., the $V_{i} d N_{i} / d t \approx 0$ ), as has been assumed in the recombination model discussed above.

The above considerations give the electron temperature in terms of measurable experimental quantities during the recombinational relaxation. It should be noted that currently available experimental techniques for directly measuring electron temperatures in a transient plasma, under the conditions of these experiments, typically do not exhibit the good accuracy desirable for recombination coefficient determinations. This circumstance is a justification for the calculation procedure that has been employed here in determining the electron temperature during the recombination process.

Data Reduction: From the directly measured quantities during the relaxation period, the electrical conductivity and the number densities in the upper states of the observed transitions may be computed as a function of time. The latter are obtained from the spectroscopically measured relative changes in population and from the measured initial conditions. These measurements also provide an estimate of the total plasma radiation loss as a function of time.

In order to determine the electron density and electron temperature, one must solve the equation for the quasi-steady energy balance on the freeelectrons given in Eq. (8) simultaneously with the expression for the nonequilibrium electrical conductivity as given in Ref. 7. A fairly accurate initial estimate of the electron density was obtained from the electrical conductivity alone, and then electron temperature and density were finally calculated by a simple iterative procedure. ${ }^{10}$

\section{Ionization}

The analysis of the ionization process following a step increase in electric field strength can be performed with a simplified physical model, as was done for the recombination process treated above.

Upon abrupt application of the electric field, the electron temperature rises rapidly and the low-lying states below the critical gap are populated relatively rapidly by electronic collisions to approach quasisteady thermal equilibrium between themselves and the ground state at the instantaneous electron temperature (when radiative depopulation effects can be ignored). This condition is defined by the relation $N_{i} / N_{1}=\left[g_{i} \exp \left(-V_{i} / k T_{\mathrm{e}}\right)\right] / g_{1}$, where $V_{i}$ is the excitation energy of the $i$ th lower level with respect to the ground state, and $N_{i}$ and $g_{i}$ and $N_{1}$ and $g_{1}$ are the number density and degeneracy of the $i$ th state and of the ground state, respectively.

Simultaneously, the closely-spaced upper energy levels located above the critical gap near the continuum tend to approach a mutual (Saha) electronic collisional equilibrium with the free electrons as defined by equations like Eq. (6). This condition is defined in terms of the instantaneous electron temperature and instantaneous electron density (not necessarily the electron density corresponding to complete thermal equilibrium at the instantaneous electron temperature).

Transitions across the critical gap occur on a relatively longer time scale than the times associated with the establishment of these quasi-steady populations, and the total rate of ionization is limited by this critical rate of upward crossings of the critical gap. Thus we have two quasi-steady "equilibrium" conditions maintained separately due to their isolation caused by the slow rate of excitation across the critical gap. Only at the end of the ionizational transient do the populations of all the excited states above and below the gap and the density of the free electrons tend to be in complete thermal equilibrium at the final electron temperature, provided radiative depopulation effects are ignored.

From the discussion of the preceding section, the total rate of ionization is given by

$$
N_{\mathrm{e}}^{-1}\left(d N_{\mathrm{e}} / d t\right)=\left.\left(\gamma / N_{\mathrm{e}}\right)(d N / d t)\right|_{u \min },
$$

where $\left.(d N / d t)\right|_{u \text { min }}$ is determined from the following relation by calculation for each energy gap $U$ :

$$
\begin{aligned}
& \left.\frac{d N}{d t}\right|_{u}=N_{\mathrm{e}}\left\{\frac{N_{1}}{g_{1}} e^{-\left(U+V_{0}-E_{k}\right) / k T_{0}}\right\} \bar{c} \sigma_{0} \\
& \cdot \sum_{i} \frac{B_{i} g_{i}}{\left(U+E_{i}-E_{k}\right)^{2}}\left(1+\frac{2 k T_{\mathrm{e}}}{U+E_{i}-E_{k}}\right),
\end{aligned}
$$

where $N_{1}$ and $g_{1}$ are the number density and degeneracy of the ground state. The factor $\gamma$ will again be regarded as having the approximate value $\frac{1}{3}$ for the conditions of the ionization experiments.

Combined Ionization and Recombination: Near the end of the relaxation transients, both ionization and recombination processes are important. The foregoing expressions for ionization and recombination rates can be combined to give the following result for the net rate of change of electron density:

$$
d N_{\mathrm{e}} / d t=\left.\gamma(d N / d t)\right|_{u \min }\left[1-\left(N_{\mathrm{e}} / N_{\mathrm{eq}}\right)^{2}\right] .
$$

Note that the importance of recombinational processes relative to ionizational processes is given by the ratio $\left(N_{\mathrm{e}} / N_{\mathrm{eq}}\right)^{2}$, where $N_{\mathrm{eq}}$ is the electron density 
Fig. 7. Electronic collisional ionization profiles for potassium.

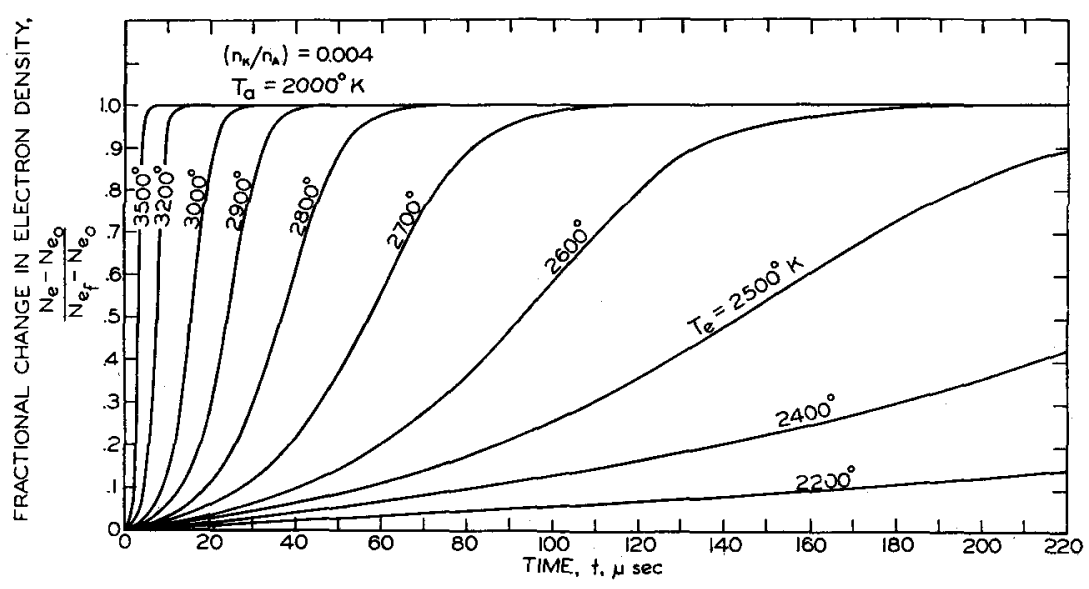

corresponding to collisional equilibrium conditions at the instantaneous electron temperature.

Electronic collisional ionization profiles have been calculated for various constant electron temperatures in the range $2200 \leq T_{\mathrm{e}} \leq 3500^{\circ} \mathrm{K}$ for the argonpotassium system and are given in Fig. 7. These calculations neglect radiative effects altogether.

Discussion of the Ionization Process: Before presenting the experimental data, a brief discussion of the transient plasma is useful. The formulation of the ionizational transient as given shows that for the dense, electron-collision dominated plasmas considered here, that ionization occurs by a multi-step process, as has also been concluded by BenDaniel. ${ }^{25}$ The Gryzinski classical cross-section expressions used here show that the probability of a collisionallyinduced transition between two neighboring atomic energy levels is inversely proportional to the square of the energy gap separating them, thus making multistep collisional processes much more probable than single-step processes. When a well-defined critical gap occurs which limits the overall rate of ionization, the correct "activation energy" for the ionization process is essentially the energy difference between the energy level immediately above the critical gap and the ground state, as is evident from Eq. (10). Neglect of this consideration can lead to large errors in the calculation of ionization rates.

As an example, for conditions of these experiments, $2000 \leq T_{\mathrm{e}} \leq 3000^{\circ} \mathrm{K}$, the energy level lying immediately above the critical gap is $0.94 \mathrm{eV}$ below the continuum, and thus the effective activation energy is $3.40 \mathrm{eV}$ instead of $4.34 \mathrm{eV}$. At $T_{\mathrm{e}}=$ $3000^{\circ} \mathrm{K}$, or $0.258 \mathrm{eV}$, the multi-step ionization rate, $\left(1 / N_{\mathrm{e}}\right)\left(d N_{\mathrm{e}} / d t\right)$, is about 40 times faster than one would calculate using only a single-step process.

${ }^{25}$ D. BenDaniel, Phys. Fluids 6, 1304 (1963).
For higher electron temperatures, the difference can be much larger, and at high enough electron temperatures $\left(T_{e} \gtrsim 5000^{\circ} \mathrm{K}\right)$, the effective activation energy is reduced to the excitation energy of the first excited state $(1.61 \mathrm{eV})$.

The ionization process following the abrupt application of an electric field to the plasma has been treated here as a sequence of events. During the first phase the electrons absorb an appreciable fraction of the power input and reach an elevated temperature determined by the quasi-steady energy balance in times that are quite short ( $3 \mu \mathrm{sec}$ for the conditions of these experiments) compared to those required for the overall ionizational relaxation. Justification of this is readily obtained by an analysis of the various terms of the energy balance of Eq. (1).$^{7,10}$ Further, during this initial phase, the populations of the excited states respond to the increased electron temperature and rapidly reach quasi-steady conditions. Experimental measurements of the $4 p$ state population of $\mathrm{K}_{\mathrm{I}}$ will be discussed below which indicates that this condition is achieved in times that are quite short compared to overall relaxation times.

After the initial phase, approximate calculations of the changes in electron temperature and density during the ionization process may be calculated by utilization of the rate expression of Eq. (11), along with the quasi-steady electronic energy balance. If we can neglect radiative losses and the energy inputs into the creation of excited state populations as a first approximation, then the quasi-steady energy balance becomes, from $\mathrm{Eq}$. (8),

$\sigma E^{2} / N_{\mathrm{e}}=\left(V_{0}+\epsilon_{\mathrm{e}}\right) N_{\mathrm{e}}^{-1}\left(d N_{\mathrm{e}} / d t\right)+\left(\dot{\Omega} / N_{\mathrm{e}}\right)$.

The omission of the $\dot{R}$ term in going from Eq. (8) to Eq. (12) will not change the results qualitatively and should be valid for large scale systems, but 


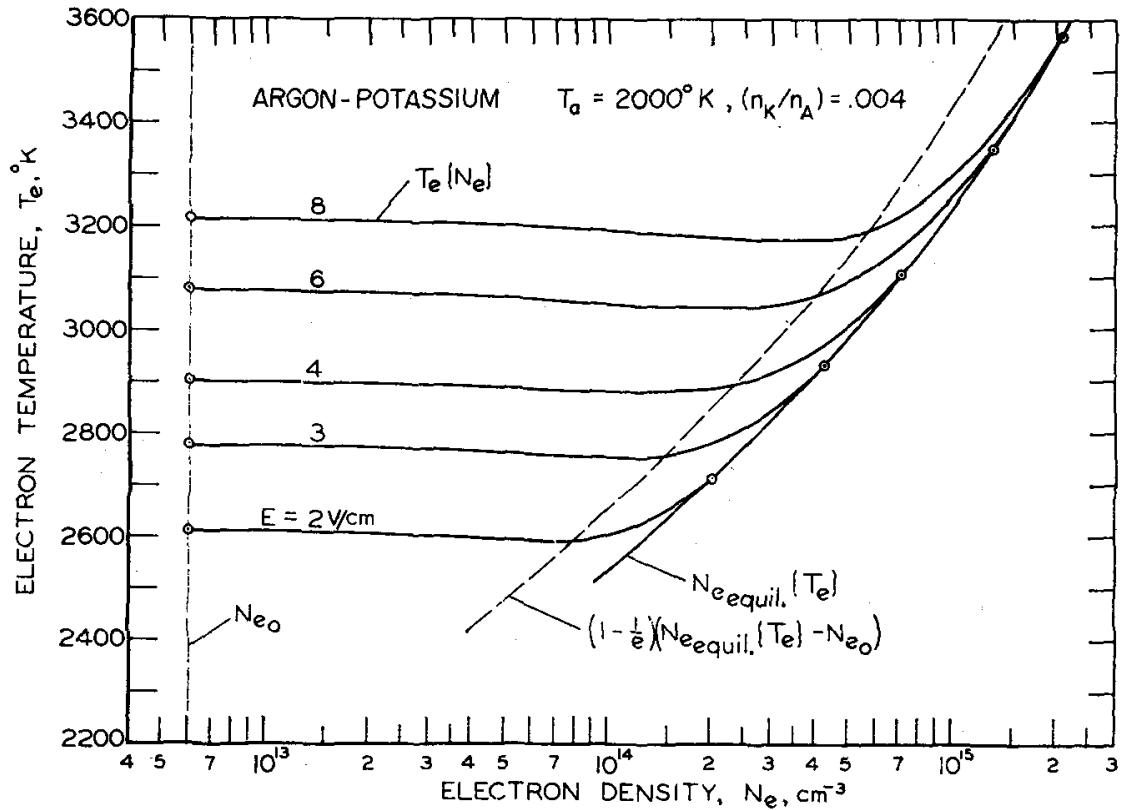

FIG. 8. Quasi-steady variation of electron temperature during ionizational relaxation. radiation losses may be important under some conditions in very small laboratory systems. The effects of radiative loss would be to reduce the electron temperature and ionization rate at a given field strength.

Calculations have been performed with Eqs. (11), (12) for typical experimental conditions in the argonpotassium plasma assuming the application of various constant electric field strengths, and the results are given in Fig. 8. Note that the electron temperature shown in Fig. 8 has a quasi-steady value which is substantially elevated above the gas temperature throughout the entire ionization process. The electron density increases about two orders of magnitude, for average field strengths, while the electron temperature remains nearly constant. During this phase, $N_{\mathrm{e}}\left\{T_{\mathrm{e}}\right\} \ll N_{\mathrm{eq}}\left\{T_{\mathrm{e}}\right\}$, where $N_{\mathrm{eq}}\left\{T_{\mathrm{e}}\right\}$ is the electron density corresponding to equilibrium at the instantaneous electron temperature $T_{\mathrm{e}}$. The rate of relaxation is determined by the instantaneous net rates of ionization during this phase which depend upon the instantaneous electron temperatures and densities as related through the quasi-steady energy balance. After the electron density has increased to about $63 \%$ of its final increase, the electron temperature increases about $10 \%$ further. During this final phase, the electron density more closely approaches equilibrium at the instantaneous electron temperature, and finally, at the end of this phase, the final electron density has the equilibrium value corresponding to the final electron temperature, $T_{\mathrm{e} \ell}$, existing at the steady state defined by Eq. (2).

\section{EXPERMMENTAL RELAXATION MEASUREMENTS}

\section{Recombination Rate Measurements}

A typical set of data for the argon-potassium system is reproduced in Fig. 9. Here, light intensity variations for potassium transitions were recorded immediately after the abrupt reduction in applied voltage from the initial steady value. Initial current densities ranged from 2 to $17 \mathrm{~A} / \mathrm{cm}^{2}$, which corresponds to field strengths of about 2.5 to $5 \mathrm{~V} / \mathrm{cm}$ and to initial electron densities from about 1.5 to $8 \times 10^{14} / \mathrm{cm}^{3}$. The final field strengths were between 0.3 and $0.8 \mathrm{~V} / \mathrm{cm}$. Measurements are shown in Fig. 9 for two consecutive tests, made for nearly identical initial discharge conditions, but for final conditions both without an applied field and with the small electric field (about $0.39 \mathrm{~V} / \mathrm{cm}$ for this case, as given by the probe voltage difference shown) necessary to monitor the conductivity transient. Disregarding the slight relative displacement of the $4 p \rightarrow 4 s$ values, reflecting the small random fluctuations in initial light intensity, the relative changes in population of the $4 p$ and $5 p$ levels appear to be nearly identical with or without the small final applied field. This indicates that the relatively small energy input to the free electrons by this field probably has no important effect upon the free electron density variation shown in Fig. 9, which has been determined from the electrical conductivity measurements by the data reduction procedure. Note the initial 4 to $10 \mu \mathrm{sec}$ lag in the decay of the first excited state $(4 p \rightarrow 4 s$ light intensity) which 


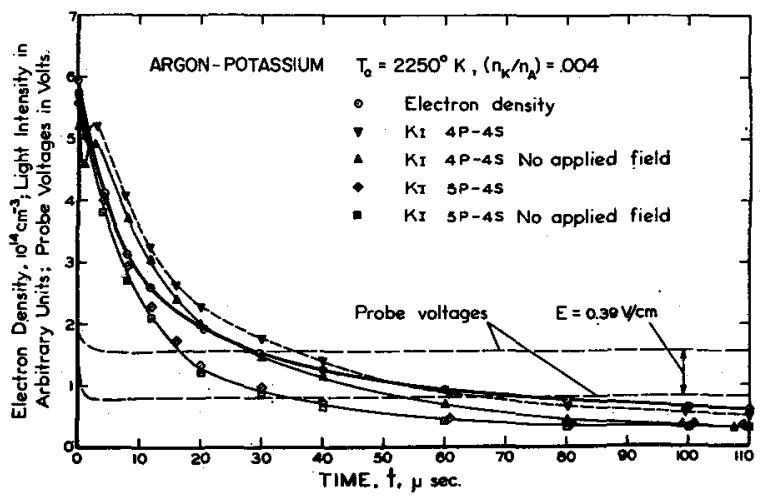

FIg. 9. Typical recombinational relaxation data.

probably reflects the initial downward cascading from higher states to the $4 p$ level.

Experimental Results: The results of data reduction calculations for a typical set of data are shown in Table I. Here, the various terms in Eq. (8) are evaluated for a number of times during the relaxation period. The last three rows of the table give the final values for the electron density and temperature, and the recombination coefficient, $\alpha=\left(-1 / N_{\mathrm{e}}^{2}\right) d N_{\mathrm{e}} / d t$. Values of the collisional recombination-rate coeffcient, $\left(-1 / N_{\mathrm{e}}^{3}\right) d N_{\mathrm{e}} / d t$, are shown in Fig. 10 as a function of the electron temperature. For all data, $n_{\mathrm{K}} / n_{\mathrm{A}}=0.004$. The data taken from the table appear as the solid circles and other data obtained from a number of different tests are also included in this figure and compared with the theory that has been discussed.

Discussion: The values of the recombination coefficient calculated for times greater than $20 \mu$ sec after the beginning of the relaxation are shown as the dark data points in Fig. 10. These data are in fair agreement with the calculated points (open circles), although they are scattered by about $60 \%$ for the range investigated. The agreement is at least as good as one would expect when considering the



FIG. 10. Collisional Iecombination-rate coefficient.

possible uncertainty in the estimated values of the electron temperatures and those uncertainties connected with the theory. In particular, note that the theoretical rates are directly proportional to the constant $\gamma$, which could easily be $50 \%$ higher or lower than the value used here. The theory is probably good to within a factor of 2 , judging by the agreement of experimental measurements in cesium with calculated values, ${ }^{23}$ and the classical cross sections used are thought to be valid to within a factor of $2{ }^{26}$ The principal uncertainties in the estimated electron temperatures are the result of possible errors in the gas temperatures.as well as errors in the electron energy balance. These possible errors give a probable uncertainty in the data themselves of about a factor of 2 .

The half-filled data points of Fig. 10 are those

TABLE I. Typical recombination data.

\begin{tabular}{|c|c|c|c|c|c|c|c|c|c|}
\hline \multicolumn{10}{|c|}{ Argon-Potassium $T_{\mathrm{a}}=2100^{\circ} \mathrm{K}\left(n_{\mathrm{K}} / n_{\mathrm{A}}\right)=0.004$} \\
\hline $\begin{aligned} 1 & \text { time, } t(\mu \mathrm{sec}) \\
2 & -1.61\left(d N_{4 p}\right) / d t \mathrm{~W} / \mathrm{cm}^{3} \\
3 & -\left(V_{0}+\frac{3}{2} k T_{\mathrm{e}}\right) d N_{\mathrm{e}} / d t \mathrm{~W} / \mathrm{cm}^{3} \\
4 & \hat{R} \mathrm{~W} / \mathrm{cm}^{3} \\
5 & \sigma E^{2} \mathrm{~W} / \mathrm{cm}^{3} \\
6 & \mathrm{\Omega} \mathrm{W} / \mathrm{cm}^{3} \\
7 & -\left(E^{*}+\frac{3}{2} k T_{\mathrm{e}}\right) d N_{\mathrm{e}} / d t \mathrm{~W} / \mathrm{cm}^{3} \\
8 & T_{\mathrm{e}}^{*}{ }^{*} \mathrm{~K} \\
9 & N_{\mathrm{e}} 10^{14} / \mathrm{cm}^{3} \\
10 & T_{\mathrm{e}}{ }^{\circ} \mathrm{K} \\
11 & \alpha 10^{-10} \mathrm{~cm}^{3} / \mathrm{sec}\end{aligned}$ & $\begin{array}{c}0 \\
\cdots \\
77.0 \\
8.4 \\
0.63 \\
69.2 \\
21.4 \\
2850 \\
5.3 \\
>3500 \\
3.64\end{array}$ & $\begin{array}{c}2 \\
\cdots 1.9 \\
8.1 \\
0.55 \\
34.4 \\
11.7 \\
2710 \\
4.0 \\
>3500 \\
3.47\end{array}$ & $\begin{array}{c}8 \\
1.0 \\
11.9 \\
5.4 \\
0.39 \\
7.9 \\
3.3 \\
2480 \\
2.27 \\
\gtrsim 3000 \\
3.05\end{array}$ & $\begin{array}{c}16 \\
0.38 \\
4.78 \\
3.06 \\
0.29 \\
2.39 \\
1.33 \\
2370 \\
1.51 \\
2580 \\
2.78\end{array}$ & $\begin{array}{r}20 \\
0.26 \\
3.25 \\
2.55 \\
0.27 \\
1.43 \\
0.91 \\
2330 \\
1.28 \\
2470 \\
2.60\end{array}$ & $\begin{array}{c}30 \\
0.11 \\
1.82 \\
1.45 \\
0.22 \\
0.70 \\
\cdots \\
\cdots \\
1.0 \\
2370 \\
2.46\end{array}$ & $\begin{array}{c}60 \\
0.03 \\
0.58 \\
0.64 \\
0.14 \\
0.11 \\
\cdots \\
\cdots \\
0.59 \\
2180 \\
2.23\end{array}$ & $\begin{array}{c}100 \\
0.02 \\
0.24 \\
0.38 \\
0.12 \\
0 \\
\cdots \\
\cdots \\
0.40 \\
2100 \\
2.06\end{array}$ & $\begin{array}{c}140 \\
0.02 \\
0.14 \\
0.32 \\
0.10 \\
-0.02 \\
\cdots \\
\cdots \\
0.32 \\
2000 \\
2.06\end{array}$ \\
\hline
\end{tabular}

${ }^{26}$ A. E. Kingston, Phys. Rev. 135, A1529 (1964). 
calculated for times as close to the time origin as possible, and in reducing this data, it has been assumed that the electron temperature is at its initial value. Though these data would not be expected to show good agreement with the calculated values, since the quasi-steady populations have not yet been established, it can be seen that fair agreement is obtained. At the other end of the temperature scale lie data obtained some $\mathbf{1 0 0} \mu \mathrm{sec}$ after the initiation of the relaxation process. Here, the electron temperatures are close enough to the gas temperatures to reduce substantially the uncertainties in electron temperature. Thus, the data for electron temperatures in the 1900 to $2300^{\circ} \mathrm{K}$ range are probably relatively more accurate.

Note that the $60 \%$ scatter in the collisional recombination rate coefficients about the theoretical values would only correspond to a $30 \%$ variation in electron density. Given this good agreement between experiment and theory, one can conclude that the electron densities as given by the two-temperature model are reasonable estimates of the actual plasma electron densities. Further, the recombination rates measured here for potassium appear to be in good agreement with present electron-electron-ion recombination rate theory.

The results given in row 10 of Table I illustrate a difficulty encountered in determining the electron temperature for times close to the time origin $(t<20$ $\mu \mathrm{sec}$ ) with the quasi-steady energy balance as written in Eq. (8). For these early times it is doubtful that a true quasi-steady condition has been yet established. Thus, the term $-\left(V_{0}+\frac{3}{2} k T_{\mathrm{e}}\right) d N_{\mathrm{e}} / d t$ is probably too high to be a valid estimate of the rate of energy input to the free electrons during this early phase, since most of the electrons will recom-
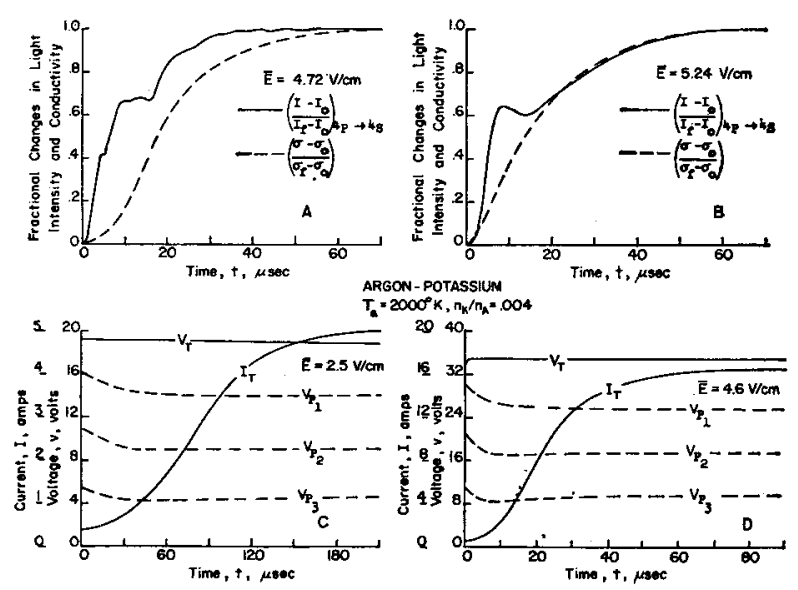

FIG. 11. Variation of light intensity and conductivity during ionizational relaxation. bine first into the uppermost levels, and then these levels must be further collisionally deexcited to the ground state. This leads to calculated values of electron temperature which are too high, even exceeding the measured initial temperature of $3000^{\circ} \mathrm{K}$ for this example as shown in row 10 of Table $I$. The average rate of energy released to the free electrons would be more adequately defined during this phase in terms of an expression of the form, $-\left(E^{*}+\frac{3}{2} k T_{\mathrm{e}}\right) d N_{\mathrm{e}} / d t$, where $E^{*}$ would be approximately equal to the binding energy of the level immediately above the critical gap. It is interesting to solve for the electron temperature during this short period at the beginning of the relaxation process by simply equating this expression to the elastic energy loss term, $\dot{\Omega}^{21}$ If $E^{*}$ is chosen to be $0.94 \mathrm{eV}$ as calculated for the range of electron temperatures between 2000 and $3000^{\circ} \mathrm{K}$, then calculated electron temperatures $T_{\text {a }}^{*}$ fall somewhat lower than before, as shown in row 8 of Table I. These temperatures look about right, since the steady-state value before relaxation is about $3000^{\circ} \mathrm{K}$. This may be regarded as somewhat fortuitous, since it is not obvious that the remaining terms in the energy balance will exactly cancel under these conditions.

\section{Ionization-Rate Measurements}

Electrical conductivities and light intensity data were recorded during the ionizational relaxation period occurring in response to the step application of an essentially constant electric field to the plasma. New data are presented here which substantiate the preliminary data presented in Ref. 7. Typical sets of observations for the argon-potassium system are shown in Figs. 11(c,d). Here, total current, total discharge voltage, and probe voltages are shown as a function of time. The voltage pulse is applied at the time origin and remains approximately constant for the duration of the test. After a short initial transient, the probe potential difference remains essentially constant. [The data in $11(\mathrm{c}, \mathrm{d})$ are better in this respect than some taken earlier. $\left.{ }^{7}\right]$ Figures $11(\mathrm{a}, \mathrm{b})$ give typical light intensity variations for the $4 p \rightarrow 4 s \mathrm{~K}_{\mathrm{I}}$ transitions and additional light intensity data is given in Refs. 7 and 10 .

Note that the behavior of the light intensity of the $4 p \rightarrow 4 s$ resonance transitions, which can be interpreted as corresponding to measured relative changes in the density of the $4 p$ state in time, indicates that the population of the $4 p$ level must rise very rapidly to a relatively high value and then reach a plateau, or perhaps decrease for a time, then finally increase relatively more slowly as the 
ionizational transient is completed. As has been discussed, when electron densities are high enough, one can relate relative changes in light intensity from resonance transitions to relative changes in the electron temperature. These relations will be valid over the latter portions of the ionizational transient; for example, the light intensity data shown in Figs. 11(a,b) can probably be interpreted in this manner back from the end of the transient up to the plateau region. An analysis of the typical data shown in Fig. 11(b) shows that the electron temperature at the plateau is within $10 \%$ of its final value, i.e., at the plateau $T_{\mathrm{e}} \approx 2920^{\circ} \mathrm{K} ; T_{\text {efinal }} \approx 3150^{\circ} \mathrm{K}$. This is a very substantial initial rise in electron temperature, and this is just the type of behavior one would expect from the theoretical considerations (see Fig. 8). This data thus gives experimental justification for the theoretical formulation of the ionizational transient. That is, the populations of low-lying states do increase very rapidly in response to a substantial initial electron temperature elevation, and that this increase in electron temperature and population density occurs much more rapidly than does the increase in current density and electron density.

It should be noted that an earlier analysis of the ionizational relaxation process was given by Kerrebrock. ${ }^{6,9}$ However, in that analysis it was assumed that for all times during the relaxation period forward and backward collisional rates are balanced, and thus that the electron density would always equal the equilibrium value at the instantaneous electron temperature. This assumption appears to be invalid as indicated by the experiments and theory presented here.

Typical measurements of electrical conductivity as a function of time, for various average applied electric field strengths in the argon-potassium system are shown in Fig. 12. Note the general similarity between these curves and the ionizational profiles of Fig. 7. The information most directly obtained from these data are the relaxation times $\tau_{\mathrm{r}}$ required for the change in electron density to reach $\left(1-e^{-1}\right)$ of the total final change, given to a very good approximation by measurements of the time required for the change in electrical conductivity $\left(\sigma-\sigma_{0}\right)$ to reach $\left(1-e^{-1}\right)$ of the final value $\left(\sigma_{\max }-\sigma_{0}\right)$.

In analyzing the data, it is convenient to give the results as a function of a characteristic time for the process. In this connection, note that in Eq. (12) for high enough field strengths, $\dot{\Omega} / N_{\mathrm{e}} \ll$ $\left[\left(V_{0}+\epsilon_{\mathrm{e}}\right) / N_{\mathrm{e}}\right]\left(d N_{\mathrm{e}} / d t\right)$, and $N_{\mathrm{e}}^{-1}\left(d N_{\mathrm{e}} / d t\right) \approx$ $\left(\sigma / V_{0}\right)\left(E^{2} / N_{\mathrm{e}}\right)$ if $\epsilon_{\mathrm{e}} \ll V_{0}$. Thus, for this case,

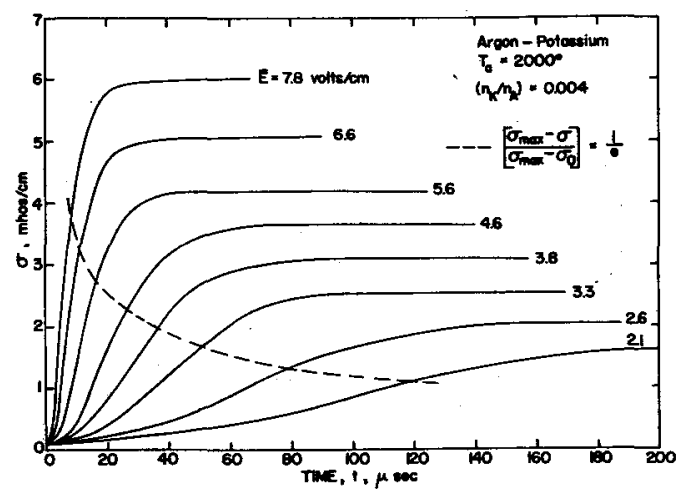

FIg. 12. Variation of conductivity with time and field strength.

the rates of ionization and energy input per electron are simply related regardless of the details of the ionization mechanism. Further, the rate of energy input per electron $\sigma E^{2} / N_{\text {e }}$ does not change rapidly during the ionizational transient for a constant $E$ since the electrical conductivity is nearly proportional to the electron density, except near the end of the transient when electron-ion interactions may be important. This discussion suggests that the group $\left(N_{\mathrm{e} 0} V_{0} / \sigma_{0} E^{2}\right)$ forms the appropriate characteristic time, except for very low field strengths where $\dot{\Omega} / N_{\text {。 }}$ is not negligible.

Values of $\tau_{\mathbf{r}}$ are given in Fig. 13 for a range of values of potassium concentration and as a function of the characteristic time, $\left(N_{\mathrm{e} 0} V_{0} / \sigma_{0} E^{2}\right)=\tau_{\mathrm{c}}$. These data include the preliminary data presented in Ref. 7 .

Comparison of Measured Ionization Rates with Theory: Equation (11) contains an expression for the rate of ionization in terms of the instantaneous electron temperature and density. Equation (12) gives the approximate simplified electronic energy balance which enables one to relate the instantaneous electron temperature and density subject to the assumptions discussed. These equations enable the determination of $T_{\mathrm{e}}=T_{\mathrm{e}}\left\{N_{\mathrm{e}}\right\}$ and thus $N_{\mathrm{eq}}\left\{N_{\mathrm{e}}\right\}$. Therefore, all the quantities in Eq. (12) can be put in terms of the instantaneous electron density, and the resulting equation can be integrated to determine $N_{\mathrm{e}}$ as a function of time. The results shown in Fig. 7 give the ionizational profiles for the constant $T_{\mathrm{e}}$ case as determined by integrating Eq. (12).

To compare experiment with theory, we assume the electric field $E$ to be characterized by a constant average value $\bar{E}$ during the ionizational transient. With this assumption, we can employ the constant $E$ results shown in Fig. 8. Note that for this case the electron temperature is so nearly constant during the first $\left(1-e^{-1}\right)$ of the ionizational transient, that 


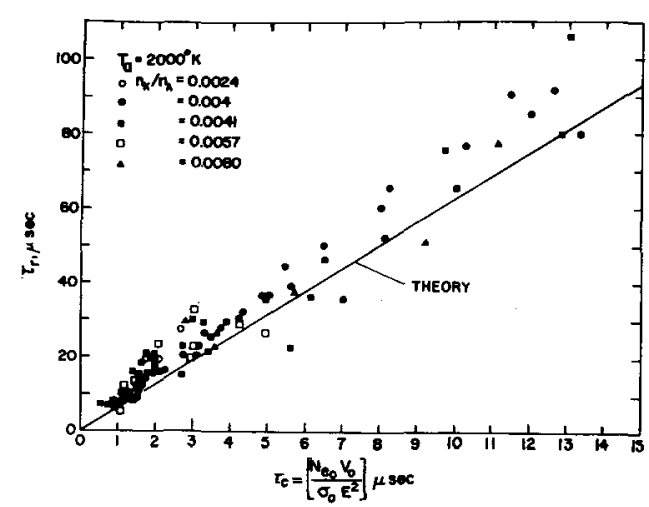

FIg. 13. Variation of relaxation time with characteristic time.

one can consider the ionizational process to occur at a constant average $T_{\mathrm{e}}$ to obtain a rough estimate of the ionization relaxation time.

For example, from Fig. 8 we see that for $\bar{E}=$ $3.0 \mathrm{~V} / \mathrm{cm}$ that $\bar{T}_{\mathrm{e}} \approx 2770^{\circ} \mathrm{K}$. Now, from Fig. 7 , we see that $\left(1-e^{-1}\right)$ of the change in $N_{\mathrm{e}}$ occurs in $\tau_{\mathrm{r}} \approx 47 \mu \mathrm{sec}$. The value of the characteristic time $\tau_{0}$ is found by separate calculation to be $\tau_{c} \approx 7.6 \mu \mathrm{sec}$ for the case considered here with $\bar{E}=3.0 \mathrm{~V} / \mathrm{cm}, n_{\mathrm{K}} / n_{\mathrm{A}}=0.004$, and $T_{\mathrm{a}}=2000^{\circ} \mathrm{K}$. In this manner the theoretical curve shown with the data of Fig. 13 was estimated.

Note that the agreement between the theory and the data shown in Fig. 13 is probably much better than one would expect in view of the approximations that have been made. Equation (9) is probably valid to within a factor of 2 , as was the case for the theoretical recombination rates. The most serious difficulties are probably those connected with the important simplifying assumptions that have been made to relate experiment and theory $(E \approx$ const $=\bar{E}$, $T_{\mathrm{o}} \approx$ const $=\bar{T}_{\mathrm{e}}, \dot{R} \approx 0$, and $\sum_{i} V_{i} d N_{i} / d t \approx 0$ ). Also, as in the recombination case, errors in electron temperature estimates cause significant errors in relaxation rate estimates.

Summary of Ionization Transient Measurements:
The approximate ionization rate theory discussed here explains, at least in a qualitative way, some important phenomena which have been observed in the ionizational transients in high-pressure gas discharges. As pointed out by BenDaniel, ${ }^{25}$ observed ionizational transients have generally been one or two orders of magnitude faster than conventional calculations based upon one-step ionization mechanisms would indicate. BenDaniel concluded that multi-step processes must be the explanation for this anomaly. This conclusion has indeed been borne out by the experiments and theory presented here. Further, the theory discussed here gives a simple means by which ionization rates may be approximately calculated in terms of an effective "activation energy" for the ionization process as given by the energy difference separating the state immediately above the critical gap from the ground state.

The qualitative behavior of the light intensity from the $4 p \rightarrow 4 s$ transitions indicates that the electron temperature does rise qualitatively in a fashion predicted by the theory. Further, the reasonable agreement between the experimentally measured relaxation times for the argon-potassium system and the simplified theory gives a rough quantitative check on the theory.

Finally, the theory and data presented here indicate that the relaxation lengths at the entrance to magnetohydrodynamic ducts are determined by the rate of ionization, and that this rate appears to be rapid enough to achieve full ionizational equilibrium over negligible short relaxation lengths for typical proposed magnetohydrodynamic operating conditions. ${ }^{7}$

\section{ACKNOWLEDGMENT}

This work was supported by the Air Force Office of Scientific Research primarily under Grant No. AF-AFOSR-160-63, and partially under Contract No. AF 49(638)-1346. 